\title{
Autour des Sonnets
}

Jacques Darras et Lachlan Mackinnon

Christophe Hausermann (éd.)

\section{CpenEdition}

\section{Journals}

Édition électronique

URL : http://journals.openedition.org/shakespeare/2855

DOI : $10.4000 /$ shakespeare.2855

ISSN : 2271-6424

Éditeur

Société Française Shakespeare

Édition imprimée

Date de publication : 1 mai 2014

Pagination : 193-211

\section{Référence électronique}

Jacques Darras et Lachlan Mackinnon, «Autour des Sonnets », Actes des congrès de la Société

française Shakespeare [En ligne], 31 | 2014, mis en ligne le 01 mai 2014, consulté le 06 mai 2019. URL http://journals.openedition.org/shakespeare/2855 ; DOI : 10.4000/shakespeare.2855 


\title{
Autour des Sonnets
}

\author{
Jacques DARRAS et Lachlan MACKINNON
}

Dominique Goy-Blanquet : Our last guests are eminent academics but first and foremost they are eminent poets and it is their longstanding conversations with Shakespeare as one poet to another as it were, they are going to share with us today.

Lachlan Mackinnon taught at Winchester College until 2011 but he is also a critic, a literary journalist and a poet who writes regularly for national press. His scholarly works include Shakespeare the Aesthete: An Exploration of Literary Theory (Palgrave), Eliot, Auden, Lowell: Aspects of the Baudelairean Inheritance (Macmillan), a biography of Elsa Triolet and four collections of poetry, one of them entitled The Coast of Bohemia, another includes several sonnets. The most recent Small Hours (Faber and Faber) was short-listed for the Forward Prize in 2010. He retired from teaching two years ago and he received a Cholmondeley Award in 2011.

Jacques Darras's poetry has also been distinguished by several awards. He was the Dean of the Faculty of languages at Amiens for fifteen years but he prefers to define himself as a poet, an essayist and translator and usually proves it by publishing volumes of each kind at one go. So last month, three books came out at once: Pieter Brueghel croise JeanJacques Rousseau sur l'A1, a translation of Blake's The Marriage of Heaven and Hell and Shakespeare's sonnets. He is also a determined European, the first non-English speaker to be invited to give the Reith Lectures on BBC in 1989. He has published a long list of essays, volumes of poetry, translations of poems by Malcolm Lowry, Walt Whitman and Ezra Pound. 
Jacques Darras : Nous venons de nous mettre d'accord il y a cinq minutes exactement, avec Lachlan. Je vais lire un papier très court, rassurez-vous, en anglais et Lachlan, de façon très élégante va lire le sien en français. Et ensuite il m’a proposé que nous fassions un échange en français également. Je m'étais préparé à ce que cela soit en anglais, mais je suis un peu soulagé, je dois le dire, surtout devant de grands spécialistes comme vous. Alors je vais lire mon texte en anglais, je vais donner un titre qui brille par sa banalité pour un angliciste et qui est le suivant : For whom the bell tolls.

Some critics wonder why there are so many poets attempting to translate Shakespeare's sonnets into French. As if they were poems of little worth. More curiously, the poets themselves are very often sceptical of their own attempt. Thus Yves Bonnefoy, in his recent Gallimard translation, is highly critical of the conventionality of the Sonnets by contrast with the freedom of the plays. The difference, he says, lies in the "free verse" of the dramas contrasting with the conventional rhyming of the poems. Now, once we have respectfully pointed to Bonnefoy that what he calls "free verse" is actually "blank verse" - a rather confusing confusion on the part of a would be anglicist - the fact remains that the Sonnets seem at times to have been composed by an altogether different hand than Shakespeare's. We shall probably never elucidate the mystery. I myself had long entertained that view, until I set to work to give my own French version. In the process I have come to the conclusion that Shakespeare may well have used two different literary forms of self-probing, with various ends and effects, one I would call metaphysical in Hamlet, the other literary or, shall we say, purely rhetorical in the Sonnets.

By definition a Sonnet is a poetic form that sounds. That is sonorous. That is based on the musical quality of words. Which is the case with Shakespeare's sonnets. They are music. Not only to my ear I guess. The easiness with which some of them are memorized comes from that very quality. Actually their musicality, their sonnetability, is precisely what makes them sound superficial. They run so smoothly, they have such gliding quality - such glibness - in them that we are too happy, when reading them aloud, not to look too closely into their meaning. 
No longer mourn for me when I am dead

Than you shall hear the surly sullen bell

Give warning to the world that I am fled

From this vile world with vilest worms to dwell.

You can hear the bell all along these lines. A sonnet is a bell ringing. Once the bell is in motion you cannot make it stop. Slow it down only. It has to exhaust itself in time. Similarly with a sonnet. There may be ten feet to that pentameter in Sonnet 71 but what you mostly hear are the strokes of the metallic bell echoing and re-echoing at the end of each line: Dead/bell/fled/dwell. I won't go further. I just wanted to make that point against Bonnefoy whose translation sounds very flat. Deliberately and wrongly so, I am afraid.

In a sonnet you have to let the bell ring itself out until you can begin to hear the premises of a meaning. Now, when coming to the meaning of Shakespeare's sonnets, one encounters a series of obstacles. Many of which are unsolvable. Let us sum them up very quickly. First, no one knows for sure who the addressees are. All the more so since they happen to change in the process. A young man (is he really the same throughout?) seems to be the poet's concern in the first 128 sonnets. A mysterious dark woman in the following 26. None having a determined identity. Which makes us all the more frustrated as we live in an age doting on biographies and who is who. The unsolvability of the identity mystery lessens the attractiveness of the Sonnets. That we cannot retell their story in novelistic prose disappoints us. Contrary to what the Romantics (Wordsworth, Coleridge) supposed, those sonnets are not autobiographical. Or if they are they are uncrackable ciphered biographies. Next comes a still larger obstacle. Deriving from the first. Is the sequence of those sonnets in the right order? And if so what does that order mean? Why should the "dark lady" come at the end and to what purpose? Does the time of the order correspond to a logic of composition or to chronological time? We shall never know, I am afraid. The sonnets are locked up. Actually they seem to have been composed on the model of the many caskets to which Shakespeare refers in his comparisons. As if they were to keep their secret for ever. Could Shakespeare have been the father of the detective poem? Playing with clueless mysteries? Rather a paradox for a playwright isn't it?

What we are left with, apart from the musical engineering of the sonnets themselves is a series of dead ends which may be their real purpose. At this stage (sorry that we have to go a bit fast) two things can 
be deduced in two directions. Either Shakespeare wanted to block any future enquiry into his identity, any raking up of his dust, as his Stratford grave says. Or his locking up all secrets in his sequence may appear as a refusal to practise the sonnet game as it used to be practised at court any further. Both explanations indeed may support each other. Originally the sonnet was an aristocratic game or pastime. Part of an accomplished education as such, on a par with the arts of war. As exemplified by Sidney, Wyatt and Surrey, all of them military strategists as well as versifiers. No such aristocratic game for William the glover's son turned playwright. In 1609 the sonnet form may well have reached its temporary exhaustion. Let us bear in mind that hardly two years later, in 1611, although there are doubts concerning that date, John Donne's Songs and Sonnets will come out. What is undisputable in Donne's case is that with him the Sonnet form is gladly mangled and torn to pieces. Donne clearly refutes the Platonic idealism that underlay the Petrarchan sonnet. He has the unmentionable human body with its sexual attributes make an irruption into the musical mould of the Sonnet. As does Shakespeare somehow in his "dark lady" sequence and its hints at venereal diseases. Donne works in the wake of Aristotle and his medical disciples. William Harvey and his discovery of blood circulation are not very far away. As a good shopkeeper Shakespeare might have wanted to close the sonnet shop before putting it on sale. Making room (with a pinch of nostalgia, possibly) for the new literary mongers or traders. For the new age demanded (let us ape Pound's Hugh Selwyn Mauberley) that all those young aristocrats so fond of wasting "love's labour" and dithering with procreation (from fairest creatures we desire increase Sonnet 1) may "exit" from the stage and be replaced. In their perverted Platonic ways they were but images of the past, narcissistic caricatures no longer at the centre of a universe of expanding trade and discovery. Their sterility being made twice obvious, what with their hatred of "increase", viz. children, (first sonnet) or their complacency for "venereal disease" (last two sonnets). In his ironical and melancholy way Shakespeare may well toll the bell for the old Petrarchan model, riddled and rifled by new medical realities (cf. the number of medical references in the last sonnets). Only Donne would through his final conversion manage to give the sonnet some respectability again, churchifying it as it were, in his Holy Sonnets. This however should by no means deter us from enjoying the musicality of the bell announcing the death of an age 
no longer to be mourned than its Stratford-born bell-ringer himself. Which is forever.

Lachlan Mackinnon : Quand je pense à vous, Jacques en tant que traducteur, je pense au traducteur d'Ezra Pound, d'Allen Ginsberg, de Walt Whitman, des poèmes longs, des formes ouvertes, et maintenant nous vous trouvons engagé avec une forme courte et fermée. Ce qui est très intéressant. Vous avez choisi d'employer des vers plus longs que ceux de Shakespeare pour la musicalité comme vous dites dans votre introduction. C'est une question permanente : pourquoi les vers français se traduisent naturellement en décasyllabes anglais mais que le décasyllabe anglais se traduit naturellement en alexandrins français ou à peu près. Vous avez parlé de l'embourgeoisement de la littérature anglaise et je crois que vous avez raison, mais je me souviens que Michael Drayton qui est issu d'un monde plus modeste que Shakespeare, a produit ses sonnets avant Shakespeare, que Samuel Daniel qui venait d'un monde un peu plus élevé a produit ses sonnets avant Shakespeare, que ce processus était en train de se passer avant la parution des sonnets de Shakespeare. Il arrive très tard dans le processus, mais il sait ce qui se passe je crois. Il est un écrivain qui s'intéresse toujours aux moments de transition. Comme Bob Dylan il s'est aperçu que « The Times They are a-Changin' » et si nous pensons par exemple au roi dans All's Well, un homme fatigué au terme de la vie vraiment, qui doit trouver un emploi pour les jeunes hommes de son royaume, la guerre, mais ce n'est pas simplement là mais c'est partout. Nous le trouvons à la fin des tragédies. En Angleterre, il y a une tradition trop longue, très bête, de parler d'un restoration of order, une restauration de l'ordre à la fin de la tragédie mais ce n'est jamais ça. C'est toujours le remplacement d'un vieux monde par un nouveau. Si nous prenons par exemple Othello : Othello lui-même est un héros d'un type ancien, qui se trouve pris au piège dans le mécanisme d'une comédie domestique où il ne sait pas se conduire. Il perd sa route et se termine magnifiquement mais son monde est passé. Macbeth se trouve dans un complot essentiellement de l'ambition de sa femme, une ambition moderne, et il est perdu. Lear, nous voyons clairement que les enfants succèdent. Avec Hamlet, nous savons bien que Fortinbras sera un meilleur roi. Hamlet est très intéressant, très engageant par sa personnalité, mais il n'est pas un roi à venir. Il ne saurait pas être roi. 
C'est un rôle trop petit pour lui vraiment. Il est habituel d'attribuer la perception des changements de l'époque à John Donne. Mais Donne, vous l'avez cité, est une personnalité très curieuse. Nous savons qu'il s'est divisé dans une lettre en Jack Donne, l'auteur des poèmes licencieux et John Donne l'auteur des Holy Sonnets. C'est une distinction fausse parce que la même personnalité sadomasochiste se montre partout.

La vie comme elle est reflétée dans les sonnets est curieuse. Une vie amoureuse qui est homosexuelle d'un côté et de l'autre mystérieuse. La tentation de lire les sonnets sous la forme d'une autobiographie à clé est très forte mais la clé est perdue. Et je crois vraiment qu'ils ne constituent pas une autobiographie. Que ce n'est pas exactement un roman, pas exactement une pièce, mais que le poète explore des situations marginales. La situation de l'homosexuel sous un régime qu'il aurait connu, comment ce serait d'être homosexuel dans un tel monde ? Question très intéressante. Question que l'on ne pouvait pas montrer à l'époque dans le théâtre mais dans des poèmes, particulièrement si les poèmes ont circulé de façon privée, et voilà un autre versant où Shakespeare se trouve : le moment où l'écrit cède à l'imprimé. L'histoire de Shakespeare, de l'œuvre imprimée est très difficile à lire. Il est probable qu'il a décidé d'imprimer les sonnets mais nous savons que Donne par exemple ne l'a pas fait. Ils ont circulé de façon privée pendant sa vie et n'ont pas été imprimés avant sa mort. Ainsi l'expérience liminale, les maladies vénériennes avec lesquelles les sonnets finissent : pourquoi ? Ce sont des questions intéressantes que vous avez examinées dans votre introduction mais peut-être il nous faut commencer avec une question linguistique. Robert Frost a dit comme vous le savez que « la poésie est ce qui est perdu dans la traduction ». Prenons un exemple :

Shall I compare thee to a Summer's day?

Thou art more lovely and more temperate:

Des vers très curieux. Cela commence très bien :

Shall I compare thee to a Summer's day?

Nous savons où nous nous trouvons : il s'agit d'un poème d'amour, un poème presque formulaïque.

Thou art more lovely 
Cela marche bien

$$
\text { and more temperate }
$$

ce mot n'est pas poétique, ce n'est pas dans le registre que le poète a établi. Le latinisme du mot, le premier mot de trois syllabes dans ces deux vers joue contre les mots courts. C'est une technique que Wordsworth, je crois, a prise en partie de Shakespeare.

Est-ce possible en français de reproduire ces transitions entre les mots courts essentiellement anglo-saxons et les longs mots latins dans une langue qui est plus latine en soi ?

Jacques Darras : C'est une question ? Bon. J'étais en train de chercher le numéro du sonnet, je devrais le savoir par cœur, mais ce n'est pas le cas,

Oserai-je te comparer à une journée d'été ?

Mais tu as plus de charme et plus de tempérance.

La tempérance c'est une vertu morale autant que climatique, même plus morale que climatique. Du moins aujourd'hui. Je ne sais pas si c'était le cas à l'époque de Shakespeare. Il faut se méfier bien entendu.

Mais la question est : qu'est-ce qu'on traduit ? L'époque de Shakespeare ou bien on traduit aujourd'hui ? Ce que j'ai essayé de faire, moi, c'est la plupart du temps d'adopter une position médiane c'est-à-dire essayer de ne pas trop m'éloigner de la langue élisabéthaine, tout en la rendant possible dans la compréhension du français contemporain. Ce n'est pas glorieux, mais ce n'est pas du tout non plus ce que font la plupart des traducteurs français qui, soit parce qu'ils ne connaissent pas l'anglais très bien, je soupçonne que c'est le cas de la majorité, soit parce qu'ils décident, ce qui est un droit du traducteur, de forcer en quelque sorte la langue, passent d'un extrême à l'autre. Ou bien ils sont très proches, trop proches jusqu'à rimer, trop proches de l'original, ce qui fait que leur langue est immanquablement aspirée par le côté élisabéthain et donc est frappée automatiquement de caducité, du moins c'est un peu ce que je sens, ou alors leur langue est tellement moderne ou tellement violente dans son interprétation, tellement fausse définitivement et délibérément, que là on se dit, Shakespeare est capable de tout supporter, la preuve, il y a eu des exemples infinis comme cette fameuse troupe belge qui lisait tout Shakespeare en quatre-vingt-huit minutes, ce 
qui était très efficace, d'ailleurs. C'est ça qui fait la force de Shakespeare, c'est qu'il est absolument indéformable. Alors moi j'ai choisi, c'est pour cela que je dis que ce n'est pas très glorieux, allez, j'ai essayé d'être subtil, c'est-à-dire de passer au milieu, de ne pas, surtout pas, trahir l'extraordinaire subtilité de ces sonnets. Nous sommes d'accord sur ce point ? Qui est une subtilité sémantique, polysémique.

Dominique Goy-Blanquet : Tu avais déjà dialogué avec Shakespeare dans William Shakespeare sur la falaise de Douvres où tu avais pris un parti tout à fait différent. Tu peux nous dire comment tu es passé de l'un à l'autre ? Et ensuite je vous laisse poursuivre votre discussion.

Jacques Darras : Je ne pense avoir répondu entièrement à la question de Lachlan mais ce n'est pas grave. C'est très simple : j'ai fait ce travail sur Shakespeare à Calais en face de la falaise par beau temps, quand la brume et le brouillard se lèvent de dessus la Manche, et il suffisait que je tourne la tête à gauche, je voyais la falaise de Douvres. Mais j'étais plus jeune de trente ans. J'étais plus jeune de trente ans donc j'étais plus près de l'Amérique, je ne voyais pas simplement l'Angleterre, je voyais aussi l'Amérique par la fenêtre de Calais. J'étais plus jeune et j'étais plus désireux d'amener du désordre. Je m'étais dit, je pense qu'il y a une certaine logique enfin dans le raisonnement : on ne peut pas traduire Shakespeare comme cela, à plat, linéairement, et je continue à le croire et à le penser mais je ne me conduis plus de la même façon, il y a un centre de gravité dans chaque sonnet, je ne sais pas si tu vois ce que je veux dire, il y a un centre de gravité et je cherchais ce centre de gravité, j'organisais ma traduction du sonnet autour de ce centre de gravité. Seulement, je me suis dit, ça va me prendre un tel temps pour trouver le centre de gravité de chacun des 154 sonnets, j'ai abandonné, j’ai laissé, j'ai quitté. Et puis, trente ans plus tard, je suis plus rangé, je suis plus en ordre, je suis plus ordonné, et j'ai fait une traduction que je crois dans l'ensemble assez correcte, mais en même temps plus posée, plus calme. Je me suis retiré dans ma maison de Stratford d'une certaine façon, un petit peu. J'espère avoir répondu.

Alors ce sonnet sur la tempérance, oui bien sûr, mais que voulais-tu montrer? 
Lachlan Mackinnon : J'ai l'impression que vous l'avez moralisé, ce sonnet.

Jacques Darras : Mais c'est très moral, d'un bout à l'autre. Dans ces sonnets-là, l'image sémantique, si je puis dire, la plus fréquente, la plus nombreuse, majoritaire c'est le juridique. Partout, tout le temps, il est question de contrat. Et c'est très intéressant parce que le premier qui ait écrit des sonnets c'est un Italien, qu'on appelle, je ne retiens jamais son nom, Giacomo da Lentini. À la cour de Frédéric II, on l'appelle le juriste, « Il Notaïo » en italien. Je crois que c'est Dante qui le désigne comme cela. C'est quand même étonnant : les quatorze vers d'un sonnet sont de toute façon un contrat. C'est un contrat que l'on passe avec le lecteur. En quatorze vers, il faut qu'il se passe quelque chose. Et il y a intérêt à ce qu'il se passe quelque chose. Et donc moi je pense que ce n'est pas étonnant que ce soient des juristes et des mathématiciens à la Cour de Frédéric II, c'étaient essentiellement des juristes et des mathématiciens, qui ont inventé le sonnet - tout est là.

En France, aujourd'hui le mathématicien poète Jacques Roubaud s'intéresse au sonnet. Il y a quelque chose de très contraignant, le maximum d'effet dans le minimum d'espace - c'est le sonnet. Ce qui n'est pas le cas de la poésie. Alors Whitman et Ginsberg c'est vraiment l'opposé. Oui mais le sonnet est la matrice de toute la poésie anglaise, par exemple, tu as mentionné Wordsworth, j'ai été très long avant de savoir - j'ai dans ma bibliothèque les Collected Works de Wordsworth, bien entendu, Tintern Abbey, Intimations of Immortality, etc... mais à la fin de sa vie, Wordsworth n'écrit que des sonnets. Combien de sonnets? Trois cents? Quatre cents?

Lachlan Mackinnon : Mais peut-être qu'il aurait-il dû cesser !

Jacques Darras : C'est vrai : il n'y en a aucun qui tienne vraiment la route hormis The world is too much with us etc.... Mais il a passé tout le reste de sa vie à en écrire quand il était à Rydal Mount, qu'il était un poète officiel, un poète établi, un poète lauréat d'ailleurs, eh bien il a écrit sonnet sur sonnet. Je réponds à ta question du début, non pas que je me compare à Wordsworth, mais dans le fond c'est un cas de régression. Je suis passé de Whitman aux sonnets de Shakespeare, c'est une régression 
mais j'ose croire quand même que c'est une régression qui revient à la source, au creuset d'origine.

Lachlan Mackinnon : Il est intéressant que vous voyiez les poètes comme des avocats.

Jacques Darras : Mais oui, il n'arrête pas de plaider, c'est une plaidoirie permanente, dans les sonnets.

Lachlan Mackinnon : J'ai une amie qui écrivait des romans policiers, elle s'appelle Sophie Hannah, je ne crois pas qu'elle soit encore publiée en France ; elle est aussi poète, bon poète et elle dit que pour elle le processus d'écrire un poème est exactement le même que celui d'écrire un roman policier. On organise tout.

Une autre question que je voulais vous poser : le poète en traducteur n'est pas le traducteur simple ; il y a toujours quelques motivations privées, ou quelque intérêt privé aussi.

Que pensez-vous avoir appris en poète dans la traduction de Shakespeare?

Jacques Darras : J'ai déjà essayé de traduire Hamlet. Ma traduction est dans un tiroir. Je n'y ai pas touché depuis vingt ans. Je ne suis pas satisfait. Je l'ai traduite en vers blancs également, un alexandrin blanc en quelque sorte, parce que ça c'est un des problèmes pour moi. C'est un des enjeux. Alors évidemment ce n'est pas un enjeu important pour les shakespeariens français ni anglais, je ne vais pas m'étaler sur cet enjeulà, mais il y a un enjeu dans la poésie française contemporaine, ou plus précisément la poésie, qui traverse tout le vingtième siècle, c'est celui de la métrique. Quel mètre employer ? La question se pose toujours à nous aujourd'hui.

Lachlan Mackinnon : Timothy Steele a publié il y a vingt-quatre ans un livre sur cette question de la métrique.

Jacques Darras : Cette question tarabuste, taraude les poètes français, qui se l'avouent ou pas, depuis le début du vingtième siècle, où la césure s'est très mal faite entre la poésie régulière baudelairienne et hugolienne et le vers libre. Alors que je trouve que l'Angleterre a inventé 
par imitation de l'Italie, grâce à quelqu'un qui s'appelle Surrey, Henry Howard, oui l'Angleterre a inventé un compromis entre le vers régulier et le vers libre. C'est là que je m'oppose violemment à Yves Bonnefoy. Ce sont des problèmes de prosodie, ce sont des problèmes importants. L'Angleterre a inventé un compromis. Ce compromis est tellement extraordinaire et tellement souple, cette constitution poétique orale est tellement formidable qu'aujourd'hui encore, beaucoup d'entre vous écrivez en pentameter blank verse.

Lachlan Mackinnon : Mais pour cela, il faut écrire dans une langue qui utilise le rythme. Normalement, le français est plus syllabique, on n'entend pas les emphases de la même façon, je crois.

Jacques Darras : Les Français pratiquent la conversation. Une des réponses que je peux donner pour ma traduction, je suis obsédé en poésie par le dialogue. J'aime que dans un poème, il y ait du dialogue. Je suis social, j'aime la conversation, j'aime beaucoup au fond les autres, et la société, je ne peux pas concevoir qu'une poésie soit uniquement tournée sur elle-même, ou sur le langage purement et simplement, comme tant de poèmes aujourd'hui. J'aime qu'il y ait de l'échange dans un poème. Et alors là, les sonnets, c'est assez extraordinaire, même si l'addressee, celui à qui s'adresse le sonnet ne répond pas physiquement, linguistiquement, mais on comprend à travers ce que dit le poète ce qu'il pense. Et donc il y a du dialogue dans les sonnets. Quatorze vers, c'est une scène, c'est un dialogue : tu ne me comprends pas, tu ne m'as pas compris, donc on reconstitue sans aucun problème cette scène. C'est un petit théâtre.

Lachlan Mackinnon : Il y a aussi les deux parties du sonnet. La volta, comme on dit en italien. Le sonnet se répond. Ainsi il contient un débat intérieur mais aussi adressé à l'autre. Je pense ceci, je pense cela, qu'estce que vous pensez?

Jacques Darras : Chez Shakespeare, il y a toujours trois personnes dans un sonnet : celui à qui on s'adresse, celui qui parle et celui qui s'interroge. Il y a une division en trois. C'est ça que je vois également. Cela complique encore la perception et la traduction.

Lachlan Mackinnon : Une autre chose que vous avez dite, c'est que vous trouvez que les sonnets quelquefois glissent, que l'œil ne s'arrête 
pas pendant qu'on les lit, qu'ils sont presque trop musicaux. Ce qu'un Anglais pourrait dire de Verlaine, comment s'engager dans cette musique ? Comment avez-vous pénétré cette difficulté ?

Jacques Darras : La musique?

Lachlan Mackinnon : Oui.

Jacques Darras : C'est une question extrêmement difficile. Je vais d'abord répondre de façon générale. J'aime beaucoup la musique, j’aime beaucoup la polyphonie. La polyphonie à l'origine, alors là je vais ramener la Picardie, c'est Josquin des Prés, c'est-à-dire le dialogue entre la voix majeure et le déchant. Le chant et le déchant. Il y a très naturellement une musique dans ces sonnets et j'y étais sensible d'une certaine manière. Je ne connais pas la musique de la Renaissance, je la connais très mal. Simplement il me semble que ceux d'entre eux que nous connaissons le mieux, Shall I compare thee to a Summer's day?, No longer mourn for me when I am dead, ce sont des cellules musicales, et c'est d'ailleurs pour cela que nous les retenons. Il y a ce côté de mémorabilité des sonnets de Shakespeare - là, c'est à moi d'interroger l'Anglais : j'ai l'impression que certains d'entre vous les savez par cœur ? Vous les connaissez par cœur.

Lachlan Mackinnon : Les sonnets comme Hamlet sont devenus le Mona Lisa de la littérature anglaise : c'est-à-dire que l'on peut les citer dans un journal sans explications. Et tout le monde sait ce que l'on veut dire. Et il y a des vers des sonnets que tout le monde reconnaîtrait. Shall I compare thee to a summer's day? , presque tout le monde le connait. Mais nous savons que la mémorabilité a été un effet recherché par les dramaturges de l'époque et tout poète cherche à être mémorable.

Jacques Darras : Oui mais, Lachlan, j’adore ce que tu dis à l'instant, ce que tu oses dire : dans le monde contemporain, un poète doit consciemment ou inconsciemment, chercher à être mémorable ou mémorisable, ce qui n'est tout à fait la même chose. Mémorisable c'est plus humble que mémorable d'une certaine façon. Et ce n'est pas le cas de beaucoup de poètes contemporains en Angleterre ou en France, et cela c'est d'une extraordinaire difficulté car il faut en plus la grâce, il faut 
un moment de grâce. Les 154 sonnets de Shakespeare ne sont pas tous mémorables mémorisables, il y en a une dizaine qui sont mémorables, qui sont absolument au-dessus, ce sont les fleurs suprêmes, et c'est très important ce que tu dis.

Lachlan Mackinnon : Mais ce n'est pas volontaire : on ne peut pas dire « Aujourd'hui je serai mémorable ».

Jacques Darras : À propos de ce sonnet, Shall I compare thee to a summer's day? je ne savais que nous allions nous attarder sur celui-ci. Ma traduction Grasset a été fugitivement présentée, excuse-moi de faire de la publicité, au Grand Journal de Canal +. Pendant dix secondes un type très doué, d'ailleurs, Trapenard, c'était le jour de la Saint Valentin, a montré très rapidement ce livre, les sonnets venaient de sortir, et il s'est tourné de façon mi-ironique mi-amusée vers le journaliste célèbre de Canal +, Jean-Michel Apathie, et il lui a dit : " Oserai-je te comparer à une journée d'été ? » et l'autre a pris cela à bout portant, et je me suis dit au moins c'est de la publicité qui est faite pour le livre. Je ne sais pas s'il connaissait Shakespeare avant mais c'est ce vers-là qu'il est allé chercher et il l'a mis en situation. C'est un vers tout à fait extraordinaire.

Lachlan Mackinnon : Nous avons parlé de la musique et de la mémorabilité. Un vers qui m'a hanté toujours, Lilies that fester, smell far worse than weeds.

Jacques Darras : Regarde, je l'ai noté. Justement je voulais en parler. Je me suis posé la question : l'auteur, l'écrivain à la mode sous Elisabeth c'est John Lyly. Je me suis posé la question il y a quinze minutes, en venant ici. Je suis allé chercher Euphues. On a l'impression que c'est de la parodie, que les sonnets de Shakespeare sont une parodie de Euphues. Une critique, une satire comme dans Love's Labours Lost. Je me demande s'il ne s'agit pas d'un traité satirique contre l'Euphuisme.

Lachlan Mackinnon : C'est bien possible. Lire Lyly c'est presque impossible. Lisons ce sonnet.

Jacques Darras : Tu me permets de lire trois lignes de Lyly? 
How frantic are those lovers which are carried away with the gay glistering of the fine face? The beauty whereof is parched with the summer's blaze and chipped with the winter's blast: which is of so short continuance, that it fadeth before one perceive it flourish.

Mais c'est Shakespeare, ça c'est un sonnet, summer's blaze, winter's blast?

Lachlan Mackinnon : Ce qui est intéressant, les méthodes que Lyly emploie, on peut les trouver aussi chez Thomas Nashe. Mais Nashe vit tandis que Lyly ne vit pas.

Jacques Darras : Je suis d'accord. On a l'impression que Shakespeare apporte de la profondeur, retourne le langage de Euphues, de Lyly en le renversant, en lui donnant une épaisseur, une profondeur que Lyly n'a pas. Mais on est dans le langage à la mode, le langage de cour sous Elisabeth. Je suis assez saisi.

Lachlan Mackinnon : Si on lit par exemple, Colin Clouts Come Home Again, de Spenser, on trouve une parodie aussi de cette poésie courtoise, cette poésie amoureuse. Shakespeare n'est pas seul. Je veux dire qu'il n'est pas le seul à s'apercevoir des limitations de ce genre.

Lisons ce sonnet :

\section{SONNET 94}

They that have power to hurt, and will do none, That do not do the thing they most do show, Who, moving others, are themselves as stone, Unmoved, cold, and to temptation slow: They rightly do inherit heaven's graces, And husband nature's riches from expense; They are the lords and owners of their faces, Others but stewards of their excellence. The summer's flower is to the summer sweet, Though to itself it only live and die, But if that flower with base infection meet,

The basest weed outbraves his dignity: For sweetest things turn sourest by their deeds; Lilies that fester smell far worse than weeds.

Jacques Darras : Avant de lire la traduction française que j’ai donnée, quelque chose me touche très profondément en traduisant, et je complète ma réponse à ta question : il y a un poète que j'adore, que 
j'aime beaucoup et qu'il m'a été permis de redécouvrir à travers beaucoup des sonnets de Shakespeare, c'est Du Bellay. En relisant Les Regrets de Du Bellay, il y a énormément de poèmes qui commencent par "Ceux qui », « Ceux qui », " They that have power to hurt and will do none » ça c'est du Du Bellay. Or il a été traduit par Spenser. On a vraiment l'impression dans certains poèmes, peut-être pourrons-nous en lire un ou deux, que c'est Du Bellay. Ce n'est pas du Du Bellay traduit, mais on entend l'écho de du Bellay, et le mécontentement de Du Bellay avec la cour de Rome, j'ai l'impression de le réentendre chez Shakespeare, mécontentement très subtil avec la cour anglaise. C'est une impression.

Ceux qui, ayant puissance de blesser, ne blessent pas,

Ne commettant pas l'acte qu'ils semblaient devoir faire,

Qui ébranlent les autres mais gardent façade de pierre,

Que rien jamais ne tente, tant ils savent rester froids :

Il est juste qu'ils héritent des cadeaux du ciel,

Qu'ils préviennent la dépense des richesses naturelles ;

De leur visage ils ont maitrise et seigneurie,

Quand les autres ne peuvent qu'en gérer l'excellence.

La fleur qui naît l'été embaume par sa fragrance,

Même si c'est pour elle seule qu'elle vit, qu'elle meurt,

Mais qu'une simple anémie malheureusement l'infecte,

L'herbe la plus vulgaire en parfum la vaudra.

Les actes peuvent rendre acides les choses les plus douces ;

Les lys en pourrissant empestent plus que l'ivraie.

Alors le Du Bellay. Est-ce qu'on peut lire le 65 ?

\section{Lachlan Mackinnon :}

Since brass, nor stone, nor earth, nor boundless sea,

But sad mortality o'er-sways their power,

How with this rage shall beauty hold a plea,

Whose action is no stronger than a flower?

O how shall summer's honey breath hold out

Against the wrackful siege of batt'ring days,

When rocks impregnable are not so stout,

Nor gates of steel so strong, but time decays?

O fearful meditation! Where, alack,

Shall time's best jewel from time's chest lie hid?

Or what strong hand can hold his swift foot back?

Or who his spoil o'er beauty can forbid?

$\mathrm{O}$, none, unless this miracle have might,

That in black ink my love may still shine bright. 


\section{Jacques Darras}

L'airain, la pierre, la terre, ni la mer infinie

En puissance ne surpassent l'horrible mortalité,

Donc par quel plaidoyer sa rage fléchirait

Sous la fragilité florale de la beauté?

Comment contrer les brèches du temps cet assaillant

Par l'haleine de miel du souffle de l'été

Quand les rocs imprenables n'ont pas même l'armement,

Quand le temps décompose les portes d'acier?

Ô pensée redoutable! Dans quel coffre cacher

La pierre précieuse du temps, qu'au temps elle soit soustraite?

Quelle main aurait la force d'arrêter son pied vif,

Qui d'empêcher qu'il pille et ravage la beauté ?

Aucun, sauf ce miracle capable d'avoir effet ;

Qu'en l'encre noire l'amour continue de briller.

Lachlan Mackinnon : Je n'en admire aucun, je n'en aime aucun. Ça arrête le poème de la même façon qu'un verdict.

Jacques Darras : Bien sûr, il y a tout d'un seul coup des brutalités, dont on n'a pas parlé mais tout le monde le sait, du distique final qui est terrible, qui est la condensation de la condensation d'une certaine façon, qu'il ne faut pas louper parce que le distique final..

Lachlan Mackinnon : ...est aussi souvent folklorique ou proverbial. Il est intéressant que quelquefois Shakespeare semble dissoudre le sonnet dans le discours général, qu'il cesse d'être individu, il devient collectif, vous me comprenez ?

Jacques Darras : Oui, Oui.

Lachlan Mackinnon : Qu'il travaille avec férocité avec la langue et qu'il la laisse aller où elle voudrait à la fin du sonnet, que le sonnet s'ouvre autant qu'il se ferme. 
Jacques Darras : C'est un art d'une telle complexité, il y a beaucoup d'inconscient, dans un modèle contraint comme est le sonnet, il y a énormément de laisser aller. Lachlan a raison d'insister là-dessus. C'est un jeu complexe de contraintes, de maximum de contraintes, et de maximum de libertés. Et je pense quand les deux sont réunis dans un même sonnet, c'est là que ça marche mais c'est très rare. Ce que je voulais dire simplement, c'est que chez Shakespeare, dans le distique final - je suis allé voir par comparaison les distiques des sonnets de Surrey qui sont très simples, très banals--- le distique, deux fois sur trois, est d'une férocité, tu as employé le mot férocité, terrible. C'est lui qui, me semble-t-il, je n'ai pas une connaissance suffisante de tous les sonnettistes de la Renaissance anglaise, c'est lui qui fait du distique final un coup d'épée. C'est la pointe.

Lachlan Mackinnon : Dans ce sonnet, il y a « That in black ink my love may still shine bright ». L'encre noire, c'est là, c'est devant nous, au moment de la lecture. Ce qui me vient à la fin de cette conversation, c'est l'importance pour le poète de lire. On pense quelquefois au poète comme une créature de fantaisie, une créature qui écrit de temps en temps mais la plupart du temps le poète lit, c'est ce qu'il doit faire pour savoir écrire.

Jacques Darras : En tout cas, le poète qui traduit, qu'est-ce qu'il doit lire ! Ah oui, bien sûr, dans le sens lire à l'auditoire. Lisons le sonnet 81 . Il est plus fort que le 71, plus méchant, plus noir, avec un jeu sur le mot " issue », d'un matérialisme funèbre. C'est l'ère des vanités qui commence.

\section{Lachlan Mackinnon :}

Or I shall live, your epitaph to make; Or you survive, when I in earth am rotten; From hence you memory death cannot take, Although in me each part will be forgotten.

Your name from hence immortal life shall have, Though I, once gone, to all the world must die; The earth can yield me but a common grave, When you entombed in men's eyes shall lie. Your monument shall be my gentle verse, Which eyes not yet created shall o'er-read, And tongues to be your being shall rehearse, When all the breathers of this world are dead. 
You still shall live, such virtue hat my pen,

Where breath most breathes, even in the mouths of men.

\section{Jacques Darras :}

Ou bien je survivrai pour faire ton épitaphe ;

Ou bien tu survivras, et moi je pourrirai ;

D’ici, jamais la mort n'arrachera ta mémoire,

Moi mon pluriel d'atomes nous serons oubliés.

Ton nom partant d'en bas deviendra immortel,

Moi, parti d'ici-bas, du monde m'effacerai ;

La terre ne m'offrira qu'une tombe commune,

$\mathrm{Au}$ su et vu de tous tu seras inhumé.

Ton monument sera le recueil de mes vers,

Leur douceur que liront des yeux non encore nés,

Des langues futures feront théâtre de ton être

Quand nos contemporains de souffle seront privés.

Car tu reprendras vie, par la force de ma plume,

Là où le souffle prend souffle, dans la bouche des humains.

Lachlan Mackinnon : J'ai pensé qu'on finirait avec un sonnet, un sonnet shakespearien, un sonnet qui se traduit, c'est-à-dire que les vers qui riment se traduisent, vous allez l'entendre, mais que les rimes sont parfois imparfaites, je le regrette mais il n'y avait rien à faire. Ce sonnet s'appelle « Le cygne », « The Swan » :

The public gardens, after the divorce,

Pour soulager, ils étaient trop modernes,

les jardins publics, après le divorce

were far too modern to relieve the pain

of the hurt loss you knew was your desert

(quand vos enfants y logeaient chez leur mère),

le mal qui suivait justement votre perte.

when your kids and your ( ?) ex were renting there. 
The earth makes nothing of your bitter gloom.

Mais, aujourd'hui, il faut s'y promener.

La terre oublie toutefois votre amertume:

Today. you walk that melancholy way

And still the swan glides gently like a queen: encore le cygne glisse doucement comme une reine.

(The Jupiter Collisions)

Propos retranscrits par Jean-Marc Peyron 